\title{
Atenção primária em saúde coletiva e enfermagem no contexto das ações e práticas de saúde: uma revisão integrativa
}

\author{
Primary health care, public health and nursing in the context of health actions and \\ practices: an integrative review
}
Atención primaria de salud, pública y enfermería en el contexto de las acciones y prácticas de salud: una revisión integrativa

\begin{abstract}
Eduarda Andrade de Oliveira ${ }^{1}$, Adriana Castro da Silva ${ }^{1}$, Michelli Domingos da Silva ${ }^{1 *}$, Railton da Silva Miranda ${ }^{1}$, Ana Carolina de Moraes Cruz ${ }^{1}$.
\end{abstract}

\section{RESUMO}

Objetivo: Descrever a forma de atuação do enfermeiro na atenção básica em saúde, bem como refletir sobre as práticas de enfermagem no contexto da atenção primária em saúde. Métodos: Trata-se de uma pesquisa de cunho exploratório, do tipo revisão integrativa da literatura. Por se tratar de pesquisa de cunho revisional, foram utilizadas as bases de dados eletrônicos por meio da Biblioteca Virtual em Saúde (BVS), aonde encontram-se outras bases de dados e periódicos inseridas, no entanto o presente trabalho usará as seguintes bases para estudo: Literatura Latino Americana e do Caribe em Ciências da Saúde (LILACS), Scientific Electronic Library Online (SCIELO), Biblioteca Virtual em Saúde Enfermagem Brasil (BDENF). Resultados: As consequências esperadas mostraram que os conflitos, dilemas e aparências relevantes do exercício do enfermeiro na APS, contribuiu com o pensamento crítico sobre o contexto de trabalho e a necessidade de articulação da categoria na construção do seu espaço profissional. Apontando para a ampliação dos limites de atuação profissional, exercendo as atividades de liderança que são tanto administrativas quanto às práticas da assistência direta ao usuário. Considerações finais: $A$ atuação do enfermeiro na APS é um campo amplo e vasto em processo de qualificação, sejam na prática clínica ou gerencial, essas práticas realizadas pelo enfermeiro na UBS são práticas administrativas e de assistência direta ao usuário.

Palavras-chave: Atenção primaria à saúde, Enfermagem, Saúde da família.

\section{ABSTRACT}

Objective: Describe the way nurses work in primary health care, as well as reflect on nursing practices in the context of primary health care. Methods: This is an exploratory research, an integrative literature review. As this is a revisionary research, electronic databases were used through the Virtual Health Library (VHL), where other databases and journals are inserted, however the present work will use the following bases for study : Latin American and Caribbean Literature in Health Sciences (LILACS), Scientific Electronic Library Online (SCIELO), Virtual Health Library Nursing Brazil (BDENF). Results: The expected consequences showed that the conflicts, dilemmas and relevant appearances of the nurse's practice in PHC, contributed to critical thinking about the work context and the need to articulate the category in the construction of their professional space. Aiming at expanding the limits of professional performance, exercising leadership activities that are both administrative and the practices of direct assistance to the user. Final considerations: The role of nurses in PHC is a broad and vast field in the qualification process, whether in clinical or managerial practice, these practices performed by nurses in the UBS are both administrative and direct user assistance practices.

Key words: Primary health care, Nursing, Family health.

${ }^{1}$ Universidade Nilton Lins (UNL), Manaus - AM. *E-mail: michelliclarinha@hotmail.com 


\section{RESUMEN}

Objetivo: Describa la forma en que las enfermeras trabajan en la atención primaria de salud, así como reflexione sobre las prácticas de enfermería en el contexto de la atención primaria de salud. Métodos: Esta es una investigación exploratoria, una revisión de literatura integradora. Como se trata de una investigación revisora, se utilizaron bases de datos electrónicas a través de la Biblioteca Virtual en Salud (BVS), donde se insertan otras bases de datos y revistas, sin embargo, el presente trabajo utilizará las siguientes bases para el estudio: Literatura Latinoamericana y del Caribe en Ciencias de la Salud (LILACS), Biblioteca Electrónica Científica en línea (SCIELO), Biblioteca Virtual en Salud de Enfermería Brasil (BDENF). Resultados: Las consecuencias esperadas mostraron que los conflictos, dilemas y aspectos relevantes de la práctica de la enfermera en APS, contribuyó al pensamiento crítico sobre el contexto laboral y la necesidad de articular la categoría en la construcción de su espacio profesional. Con el objetivo de ampliar los límites del desempeño profesional, ejerciendo actividades de liderazgo que sean administrativas y prácticas de asistencia directa al usuario. Consideraciones finales: El papel de las enfermeras en la APS es un campo amplio y vasto en el proceso de calificación, ya sea en la práctica clínica o administrativa, estas prácticas realizadas por las enfermeras en UBS son prácticas administrativas y asistencia directa al usuario. ejercer actividades de liderazgo que sean prácticas administrativas y de asistencia directa al usuario.

Palabras clave: Atención primaria de salud, Enfermería, Salud de la familia.

\section{INTRODUÇÃO}

A presença do enfermeiro demonstra-se fundamental para a consolidação da Estratégia Saúde da Família (ESF), contribuindo com suas variadas atribuições, desde a organização gerencial das atividades da ESF, o funcionamento do centro de saúde até o cuidado direto aos indivíduos, família e comunidade.

O enfermeiro da Saúde Coletiva descreve sua prática em diferentes áreas, como acolhimento de enfermagem individual/coletiva, atuações educativas, coordenação de cargos técnicos da vigilância epidemiológica, ainda as ações relativas à administração da equipe de enfermagem e participação com a equipe de saúde no planejamento, coordenação e estimativa das ações de saúde (KAHL C, et al., 2018).

A enfermagem deve ser respeitada como o objeto e a essência da enfermagem. Eles recomendam que a profissão do enfermeiro envolve vários saberes, entre eles, o saber afetivo, também delineado como a arte de cuidar. Segundo eles as intervenções concretizadas pelo enfermeiro se caracterizam como o cuidado no momento em que comportamentos de cuidar sejam exibidos. (ACIOLI S, et al., 2014).

As Unidade Básicas de Saúde (UBS), são consideradas como porta de entrada dos usuários no sistema de saúde, onde este espaço em que o enfermeiro é importante integrante da equipe multiprofissional da Estratégia Saúde da Família (ESF).

Em 2018 o Sistema Único de Saúde (SUS), completou 30 anos de sua existência. O Brasil é o único país do mundo que tem um sistema público de saúde regularizado no princípio da universalidade, da equidade e da integralidade. Entretanto os obstáculos enfrentados pelo SUS vêm, ao longo dessas três décadas, tornando-se cada dia mais indispensável na vida da sociedade brasileira (DUARTE E, et al., 2018).

Macinko J e Harris MJ (2015); relata que $80 \%$ da população, é exclusivamente do SUS e necessita do programa de saúde público para realização de consultas e exames relacionadas assistência básica. O Brasil atualmente, mostrou um porcentual de $11 \%$ no desemprego, aonde a sua economia está ativa e crescimento econômico é promissor para o país que vem passando por algumas dificuldades financeira na saúde pública.

A finalidade do SUS é promover o acesso direto aos pacientes dando-lhe garantias no atendimento universal e promovendo a equidade. Ainda assim, o SUS trabalha com as unidades privadas que irá prestar alguns serviços aos seus pacientes, principalmente aqueles que necessitam de uma de alta complexidade, e dos medicamentos de alto custo. Sabemos que o Governo Federal também vai disponibilizar algumas medicações de alto custo para o paciente poder dar continuidade ao seu tratamento (STOPA SR, et al.,2017). 
Doniec K, et al. (2016); comenta que o SUS é universal, e necessita do apoio em conjunto com a vigilância sanitária, para levar uma assistência de qualidade e priorizar algumas ações de saúde necessária. $\mathrm{O}$ enfermeiro tem que priorizar algumas ações como exemplo o programa nacional de imunizações (PNI), que tem a finalidade de levar imunizações para população independente da classe social do indivíduo. Mesmo com os avanços a desigualdade social ainda persiste e não conseguimos atingir $100 \%$ da população imunizadas.

Importante salientar que essas ações preventivas, além de trazer inúmeros benefícios para os pacientes, contribuem também para os cofres públicos, já que as chances de cura ficam próximas de $100 \%$. Alguns estudos relatam a dificuldade de procurar esses serviços de saúde nos horários e dias disponibilizados pelas unidades de saúde se tornando um obstáculo, especialmente na atualidade, pois as suas ocupações são justamente nos horários estabelecidos pelas unidades de saúde. No Brasil as unidades básicas de saúde apresentam algumas UBS's, com horário de funcionamento ampliado, ou seja, até as 21 horas e funciona aos sábados (ANJOS KF, et al., 2013).

A pesquisa tem como objetivo descrever e revisar na literatura sobre a forma de atuação do enfermeiro na atenção básica em saúde, bem como refletir sobre as práticas de enfermagem no contexto da atenção primária em saúde.

\section{MÉTODOS}

Trata-se de uma pesquisa de cunho exploratório, do tipo revisão integrativa da literatura. Por se tratar de pesquisa de cunho revisional, foram utilizadas as bases de dados eletrônicos por meio da Biblioteca Virtual em Saúde (BVS), aonde encontram-se outras bases de dados e periódicos inseridas, no entanto o presente trabalho usará as seguintes bases para estudo: Literatura Latino Americana e do Caribe em Ciências da Saúde (LILACS), Scientific Electronic Library Online (SCIELO), Biblioteca Virtual em Saúde Enfermagem Brasil (BDENF). Critérios de inclusão, idiomas português e inglês, gratuitos a partir de 2013, texto completo. Critérios de exclusão, outros idiomas pagos antes de 2013, resumos (Figura 1).

Figura 1 - Critérios de Elegibilidade da Pesquisa.

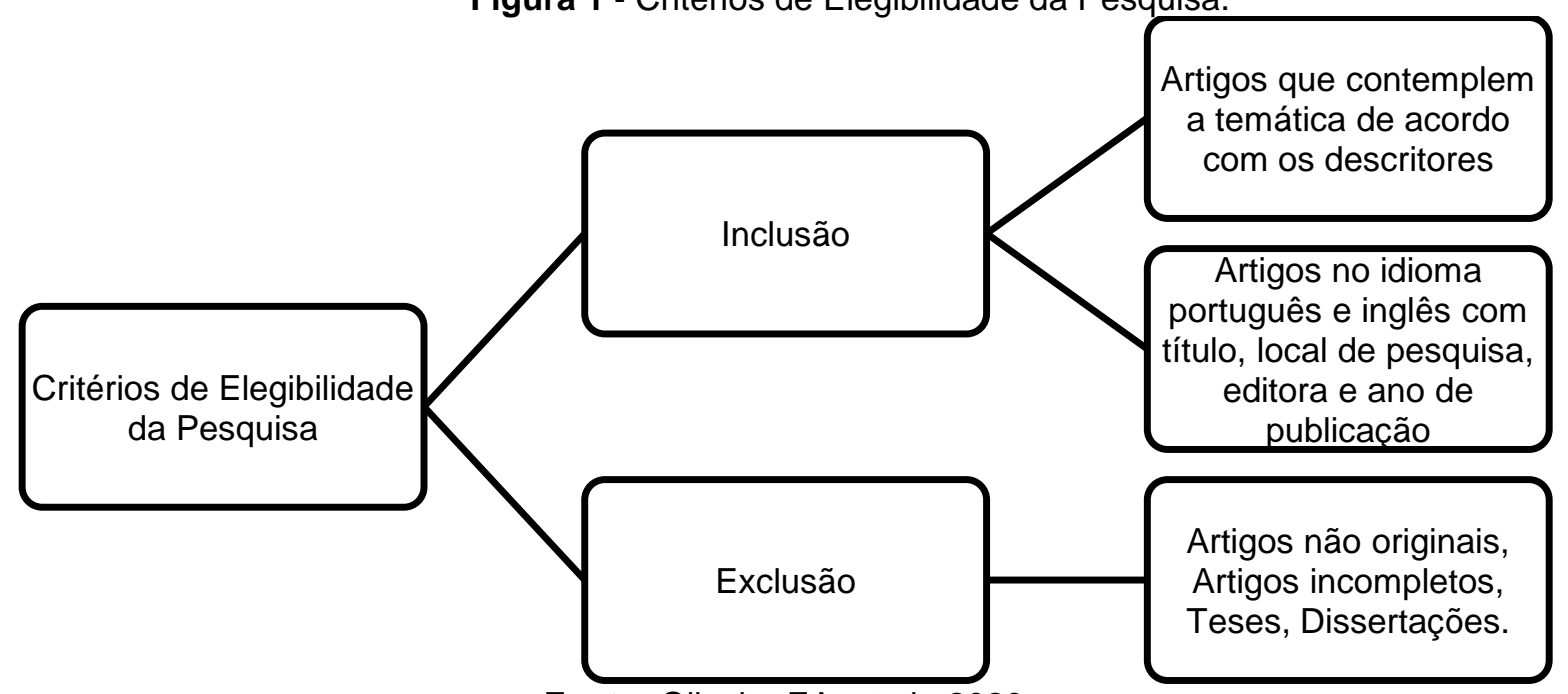

Fonte: Oliveira EA, et al., 2020.

O período de pesquisa de tais artigos se deu no período de janeiro a julho de 2020. Os descritores em saúde utilizados na pesquisa são: Atenção primária à saúde; Enfermagem; Saúde da família. Para a coleta e análise dos dados, houve um processo e fluxo para que se concentrassem artigos relativos à temática principal da pesquisa e que se evidenciasse os achados. Foram realizadas a síntese dos dados de identificação e caracterização da amostra através do instrumento de coleta de dados, com a utilização da estatística descritiva simples (distribuição de frequência) para ano de publicação, método empregado, autores, revista e bases de dados dos artigos incluídos no estudo (Quadro 1). 
Quadro 1 - Fases e descrição do percurso metodológico para qualificação de artigos.

\begin{tabular}{|c|c|}
\hline Fases da Pesquisa & Descrição \\
\hline 1a Fase & $\begin{array}{c}\text { Desenvolvimento das questões que norteiam a pesquisa e criação dos objetivos } \\
\text { específicos. }\end{array}$ \\
\hline $2^{\underline{a}}$ Fase & $\begin{array}{c}\text { Delineamento do percurso metodológico para se qualificar as evidências dos } \\
\text { artigos. }\end{array}$ \\
\hline $3^{\text {a }}$ Fase & $\begin{array}{l}\text { Busca de artigos nas bases de dados em comum com as diretrizes dos critérios } \\
\text { de elegibilidade da pesquisa. }\end{array}$ \\
\hline $4^{\mathrm{a}}$ Fase & $\begin{array}{l}\text { Análise crítica dos estudos com a organização das características principais do } \\
\text { mesmo, evidenciando a Enfermagem Baseada em Evidências. }\end{array}$ \\
\hline $5^{\mathrm{a}}$ Fase & $\begin{array}{l}\text { Síntese de dados e discussão de acordo com a interpretação técnica dos } \\
\text { resultados, comparando- com os artigos referenciados no marco teórico. }\end{array}$ \\
\hline
\end{tabular}

Fonte: Oliveira EA, et al., 2020.

\section{RESULTADOS}

Os resultados deste estudo foram baseados em artigos científicos publicados entre os anos 2018 a 2020, utilizando como descritores de saúde: Atenção Primaria à Saúde; Enfermagem; saúde da família. Para proporcionar maior objetividade ao presente estudo, foram analisados 342 artigos científicos sendo 42 em BDENF, LILACS 100 e 200 SCIELO. Após uma seleção criteriosa dos artigos restaram 2 BDENF, LILACS 1 e 2 SCIELO. Amostra final de cinco estudos $(n=5)$ referente ao tema do projeto está de acordo com (Figura 2).

Figura 2 - Fluxograma dos artigos obtidos através de bases de dados, selecionados a partir das palavras-chave: Atenção Primaria à Saúde; Enfermagem; Saúde da Família.

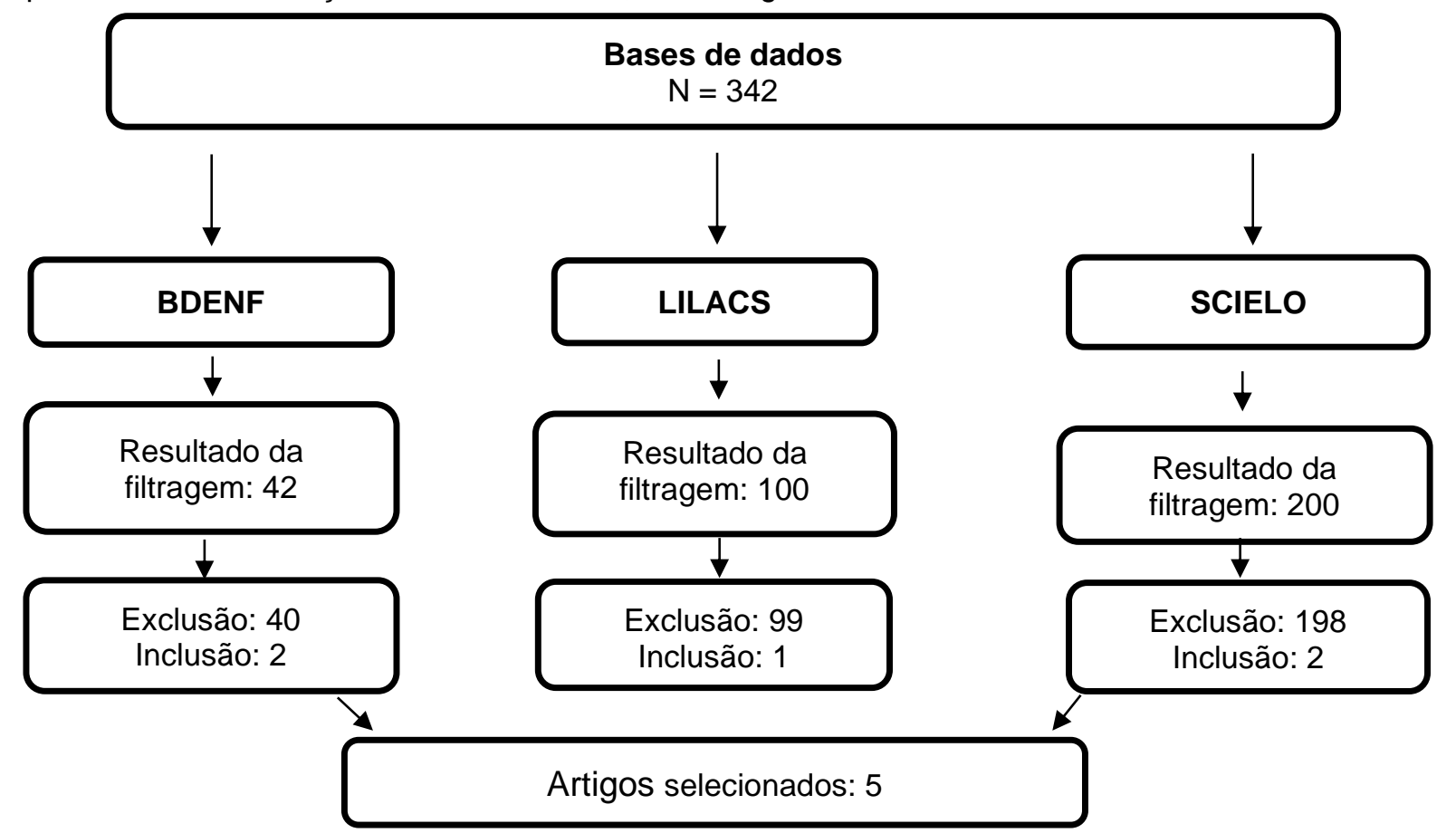

Fonte: Oliveira EA, et al., 2020.

Após a busca, a pesquisa resultou em cinco artigos para avaliação dos resultados, no formato de quadro contendo: Título, Autor/Ano, Idioma, Tipo de Estudo e Resultados. Após análise dos artigos propostas, consentiram em incluir a atuação do enfermeiro no ponto de vista do cuidado integral, por meio da coordenação de enfermagem e de atribuições pontuais da equipe de saúde. No entanto, esses "cuidados prestados pela Enfermagem", demonstrar a competência do enfermeiro de identificar necessidades de saúde da comunidade e realizar a integração que leva ao trabalho das equipes multidisciplinares, com destaque para a comunicação e a articulação (OLIVEIRA EA, et al., 2020). 
Quadro 2 - Análise de artigos sobre a atenção primaria no período de 2016 - 2020.

\begin{tabular}{|c|c|c|c|c|c|c|}
\hline $\mathbf{N}^{\circ}$ & Título & Autores/Ano & Idioma & $\begin{array}{l}\text { Tipo De } \\
\text { Estudo }\end{array}$ & Objetivos & Resultados \\
\hline 1 & $\begin{array}{l}\text { Enfermagem em } \\
\text { Saúde Coletiva: } \\
\text { desejos e práticas }\end{array}$ & $\begin{array}{l}\text { Fortuna CM, } \\
\text { et al., } 2019 .\end{array}$ & $\begin{array}{l}\text { Português } \\
\text { e Inglês }\end{array}$ & $\begin{array}{l}\text { Estudo } \\
\text { Reflexivo }\end{array}$ & $\begin{array}{l}\text { Dialogar e refletir sobre as } \\
\text { práticas da Enfermagem na } \\
\text { Saúde Coletiva, trazendo o } \\
\text { vivenciado por enfermeiras no } \\
\text { mundo do trabalho. }\end{array}$ & $\begin{array}{l}\text { Estudos realizados em grupo de pesquisa, discussões em evento científico e } \\
\text { experiências profissionais têm reforçado a importância de se reconhecerem } \\
\text { a intencionalidade do trabalho em saúde, a compreensão do processo } \\
\text { saúde-doença-cuidado que se tem e se defende, o papel dos determinantes } \\
\text { sociais e de todo o contexto histórico, político, econômico e social na } \\
\text { formação profissional, na organização dos serviços de saúde e da } \\
\text { sociedade. }\end{array}$ \\
\hline 2 & $\begin{array}{l}\text { A complexidade } \\
\text { do trabalho do } \\
\text { enfermeiro na } \\
\text { Atenção Primária } \\
\quad \text { à Saúde }\end{array}$ & $\begin{array}{l}\text { Ferreira } \\
\text { SRS, et al., } \\
2018 .\end{array}$ & $\begin{array}{l}\text { Português } \\
\text { e Inglês }\end{array}$ & $\begin{array}{l}\text { Estudo } \\
\text { Reflexivo }\end{array}$ & $\begin{array}{c}\text { Promover reflexão sobre o } \\
\text { trabalho do enfermeiro na } \\
\text { Atenção Primária à Saúde } \\
\text { (APS) e sobre os aspectos } \\
\text { necessários para a } \\
\text { (re)construção dessa prática } \\
\text { profissional, consolidando esse } \\
\text { espaço de atuação no cuidado } \\
\text { das pessoas, famílias e } \\
\text { comunidades. }\end{array}$ & $\begin{array}{c}\text { Apresentam-se conflitos, dilemas e aspectos relevantes da prática do } \\
\text { enfermeiro na APS, contribuindo com o pensamento crítico sobre o contexto } \\
\text { de trabalho e a necessidade de articulação da categoria na construção do } \\
\text { seu espaço profissional. }\end{array}$ \\
\hline 3 & $\begin{array}{l}\text { Desempenho de } \\
\text { enfermeiros no } \\
\text { serviço de } \\
\text { gerenciamento de } \\
\text { leitos de um } \\
\text { hospital de ensino } \\
\end{array}$ & $\begin{array}{l}\text { BORGES F, } \\
\text { et al., } 2020 .\end{array}$ & $\begin{array}{l}\text { Português } \\
\text { e Inglês }\end{array}$ & $\begin{array}{l}\text { Estudo } \\
\text { Descritivo e } \\
\text { Qualitativo. }\end{array}$ & $\begin{array}{l}\text { Analisar o desempenho de } \\
\text { enfermeiros no serviço de } \\
\text { gerenciamento de camas de } \\
\text { um hospital público de ensino. }\end{array}$ & $\begin{array}{c}\text { O desempenho dos enfermeiros foi descrito em três matrizes de análise, } \\
\text { representadas pelas categorias: aquisição, implementação e manutenção } \\
\text { dos recursos de enfermagem; transformação dos recursos de enfermagem } \\
\text { em serviço de enfermagem e resultados do trabalho do enfermeiro na } \\
\text { gerência do leito. }\end{array}$ \\
\hline 4 & $\begin{array}{l}\text { Atuação do } \\
\text { enfermeiro na } \\
\text { atenção primária à } \\
\text { saúde na temática } \\
\text { do câncer: do real } \\
\text { ao ideal }\end{array}$ & $\begin{array}{l}\text { Nogueira IS, } \\
\text { et al., } 2020 .\end{array}$ & $\begin{array}{c}\text { Português } \\
\text { e Inglês }\end{array}$ & $\begin{array}{c}\text { Revisão } \\
\text { integrativa } \\
\text { da literatura }\end{array}$ & $\begin{array}{l}\text { Identificar na } \\
\text { literatura brasileira atuação do } \\
\text { profissional enfermeiro } \\
\text { na Atenção Primária à } \\
\text { Saúde na temática do câncer. }\end{array}$ & $\begin{array}{c}\text { Oito estudos abordaram a realização de atividades assistenciais, como a } \\
\text { realização de consultas de enfermagem com foco em exames preventivos } \\
\text { do câncer de colo de útero e mama, atividades educativas e visitas } \\
\text { domiciliares. }\end{array}$ \\
\hline 5 & $\begin{array}{l}\text { Mental health } \\
\text { needs of } \\
\text { adolescents and } \\
\text { the nursing cares: } \\
\text { integrative review }\end{array}$ & $\begin{array}{l}\text { Teixeira LA, } \\
\text { et al., } 2020 .\end{array}$ & Inglês & $\begin{array}{l}\text { Revisão } \\
\text { Integrativa } \\
\text { da } \\
\text { Literatura. }\end{array}$ & $\begin{array}{l}\text { Sistematizar } \\
\text { o conhecimento produzido } \\
\text { acerca da atuação } \\
\text { da enfermagem diante } \\
\text { das necessidades de saúde } \\
\text { mental dos adolescentes. }\end{array}$ & $\begin{array}{l}\text { A amostra foi composta por artigos predominantemente em inglês e } \\
\text { publicados entre o período de } 1999 \text { e } 2018 \text {. As } \\
\text { principais necessidades de saúde mental dos adolescentes estão } \\
\text { relacionadas à depressão, ansiedade, estresse, uso e dependência de } \\
\text { drogas, distúrbios alimentares, dentre outras. }\end{array}$ \\
\hline
\end{tabular}

Fonte: Oliveira EA, et al., 2020. 


\section{DISCUSSÃO}

Para Acioli S, et al. (2014), descreve que o Ministério da Saúde considera as UBS a instância prioritária e a porta de entrada para acesso dos usuários ao SUS. Sabemos que nas UBS o enfermeiro é o profissional que constrói relações de diálogos, praticando a escuta ativa, a humanização e o respeito. Já para Anjos KF, et al. (2013); a sabedoria da população e a da enfermagem não são opostas e devem integrar-se, pois dessa maneira reflete na melhoria da qualidade de vida dos usuários.

A Constituição Federal de 1988, comenta que o SUS é um patrimônio nacional, onde ele conduzirá a garantia na saúde, alimentação, educação, saneamento, essa cobertura nas ações de saúde pública será financiada exclusivamente pelo do Governo Federal, junto aos Estados e Municípios que também iram contribuir para o aperfeiçoamento dos serviços ofertados pelo SUS, que tem um importante papel em prol do fortalecimento do sistema de saúde brasileiro (FERTONANI HP, et al., 2015).

Proteger o SUS é dever de todos os governos, gestores, sociedade civil organizada, comunidade científica e profissionais de saúde. Diante disso, a PNAB fortaleceu o seu proposito e a sua concepção da $A B$ atingindo as atribuições da atenção primária à saúde abrangente, reconheceu a Saúde da Família como protótipo substitutivo e de reorganização da $A B$ onde a mesma, teria sido desenvolvida e associada junto a população visando os direitos preferencial dos usuários, e sendo a sua principal porta de entrada e centro de comunicação da Rede de Atenção à Saúde (RAS) (MOROSINI MVGC, et al., 2018).

Bezerra EAF e Almeida JJJ (2014), comenta que os problemas encontrados pelas equipes de saúde, são diferentes dos serviços encontrados e relacionados à instalação e comportamento das famílias, como a falta de adesão da mesma aos tratamentos, seja este referente ao tratamento medicamentoso ou às mudanças de hábitos. Essa busca desenfreada pode resultar em processos de estresse, assuntos mal resolvidos e pessoas infelizes na resolução de sua consulta, não saber comunicar-se com todos os membros da família, também se enquadra como obstáculo.

As RAS, é o resultado esperado sobre à situação de saúde vigente em nosso país e implicam estabelecer, de formato integrada, sob organização da APS, os pontos de atenção ambulatoriais e hospitalares secundários e terciários, aos sistemas de apoio tal como: sistema de assistência farmacêutica, sistema de apoio exame de saúde e terapêutico e sistema de informação), os sistemas logísticos (sistema de regulação da atenção, prontuário eletrônico em saúde e sistema de transporte em saúde) e o sistema de governança todos os sistemas estão interligados com os Municípios, Estado e Federal (MENDES EV, 2013).

Conforme Viegas SMF e Penna CMM (2013), a integralidade estabelece um elemento central para a consolidação de um modelo de saúde que incorpore, de forma mais efetiva, a universalidade e a equidade durante $o$ atendimento às pessoas para alcançar ações resolutivas em saúde. Esses princípios se encontram fundamentada no SUS onde foram defendidos como um princípio único: o do direito à saúde. De acordo com a Lei 8080/1990 o conceito de integralidade da assistência é definido como conjunto articulado e contínuo das ações e serviços preventivos e curativos, individuais e coletivos, exigidos para cada caso em todos os níveis de complexidade do sistema.

Segundo Silva S e Carmo F (2016), explana que a integralidade pode ser alcançada a partir de vários ângulos não excludentes entre si, mas que sobressaem aos aspectos diversos da mesma demanda. Em uma primeira aproximação, a integralidade pode ser entendida como atendimento integral, focando as atividades preventivas e de promoção da saúde, sem estragos dos serviços assistenciais. Sendo, uma articulação entre promoção, prevenção e recuperação da saúde por meios de ações que se estruturam em um mesmo espaço com a constituição de saberes e ações que se completam.

Acredita-se que o primeiro desafio para a exercício essencial do princípio da integralidade é a competência dos especialistas em apreciarem o serviço que está suposta articulação entre as ofertas de ações preventivas de forma automática significando a criação de dispositivos e procedimentos coletivos além das ações preventivas demandadas pela própria população (ARANTES LJ, et al., 2016).

Negrini LDO, et al. (2017), diz que outra habilidade, além da prática sanitária, é a de planejar ações. Durante os cursos de graduação em saúde há maior direcionamento para a prestação da assistência individual e para o seguimento de protocolos. É preciso criar espaços e oportunidades de discussão para o 
treinamento das habilidades de liderança, de articulação do trabalho em equipe, de comunicação e de intervenção nas necessidades de saúde além das práticas curativas. Diante disto o enfermeiro complementa as atividades, juntamente com as equipes multiprofissionais, ao realizarem o trabalho educativo enfrentam diversas dificuldades para sua concretização (SILVA SH, et al., 2020).

Oliveira SA, et al. (2017), listaram as principais destas ferramentas: Teorias administrativas, o processo de trabalho, a ética no gerenciamento, os saberes sobre cultura e poder organizacional, qualidade de vida no trabalho, saúde do trabalhador, gerenciamento de pessoas, dimensionamento de pessoal, gerenciamento de recursos matérias, custos, recursos físicos, sistema de informação e processo decisório, a capacidade de negociação, a capacidade de negociação e a disposição para o trabalho em equipe.

Soler ZASG (2018), afirmam que o profissional enfermagem deve utilizar a Sistematização da Assistência em Enfermagem (SAE) para explanar suas atribuições em seu gerenciamento expondo as etapas nas quais busca chegar a um objetivo para aumentar o desempenho do seu ofício como líder/ gestor, garantindo uma tomada de decisão com mais assertiva a ele mesmo, à equipe e ao cliente, o alvo principal de suas metas traçadas.

Camargo RAA, et al. (2013), relata que no trabalho na ESF, o enfermeiro é responsável por implantar os programas e políticas de saúde do governo: atenção à saúde da criança, da mulher, do idoso, Hiperdia (sistema de cadastramento e acompanhamento de hipertensos e diabéticos), controle de tuberculose, eliminação da hanseníase, ações de saúde bucal, Programa Saúde na Escola (PSE), Sistema de Informações da Atenção Básica (SIAB), Núcleo de Apoio à Saúde da Família (NASF), Sistema de Informação de Agravos de Notificação (SINAN), Sistema de Acompanhamento da Gestante (SISPRENATAL), Gerenciador de Ambiente Laboratorial (GAL), Sistema de Apoio ao Relatório de Gestão (SARGSUS), Programa das Ações de Vigilância em Saúde (PAVS).

Paula M, et al. (2014), afirma que as ações gerenciais do enfermeiro na ESF envolvem coordenação e reuniões com equipe, educação continuada, capacitações, preenchimento de relatórios, planejamento de atividades, organização e administração de materiais, coordenação/gerência da ESF, supervisão dos ACS. As funções gerenciais fazem parte da rotina do enfermeiro, assim como as funções assistenciais, uma vez que ambas estão interligadas.

Melo PMJ (2018), ressalta as ações do enfermeiro na ESF constituem-se no monitoramento das condições de saúde como núcleo da atenção de enfermagem, seja no atendimento individual, seja no grupal; no levantamento e monitoramento de problemas de saúde (no enfoque de risco ou de vulnerabilidade) sendo que estes deverão estar articulados à intervenção nos agravos de ordem patológica (pautados no saber da clínica) e no exercício de uma prática de enfermagem comunicativa.

Roecker S, et al. (2013), afirma que a ação educativa do enfermeiro em saúde refere-se às atividades voltadas ao desenvolvimento de capacidades individuais e coletivas visando à melhoria da qualidade de vida e saúde. De acordo com Melo PMJ (2018); dentre as estratégias de educação em saúde, destacam-se as ações de dinâmicas de grupo, equipe de trabalho e usuários, capacitações permanentes para a equipe, reuniões e palestras em escolas, campanhas de esclarecimentos de determinadas patologias.

Kebian LVA e Acioli AS (2014), fala que as principais dificuldades encontradas na ESF referem-se à estrutura física, à falta de um veículo disponível para a unidade e dos demais profissionais de saúde (médico, enfermeiro, técnico em enfermagem e odontólogo), número insuficiente de fichas distribuídas na Unidade, falta de medicamento e de segurança no posto e inviabilidade do horário de atendimento clínico, pois há dias em que se atende por somente um turno.

De acordo com Ferreira SRS, et al. (2018), o enfermeiro representa um profissional diferenciado na atenção primária, pois possui um conjunto de habilidades únicas onde proporciona a incorporação de atividades de prevenção e promoção da saúde. Neste sentido, além das atividades assistências o enfermeiro articula atividades de diversos outros profissionais. Firmino AA, et al. (2016), afirma que competem ao enfermeiro atividades assistenciais, gerenciais, participação no processo de territorialização e cadastramento de famílias, realizar ações de cuidado a comunidade e à população adscrito, participarem do acolhimento, realizar busca ativa de casos, notificação e investigação de casos, realizar reuniões, participar do gerenciamento de insumos e materiais, realizar ações educativas, dentre outros. 
Cervi GH (2016), afirma que os profissionais de enfermagem necessitam aprimorar as seguintes habilidades: Habilidade Técnica: constitui-se a partir da experiência profissional, do estudo de protocolos e diretrizes institucionais, da discussão com colegas de trabalho, da participação em capacitações e estudos pessoais; Habilidade Humana: é a capacidade de lidar de forma harmônica com as pessoas com quem se relaciona profissionalmente; Habilidade Conceitual: constitui-se na capacidade para lidar com ideias e conceitos abstratos e está ligada a pensar, raciocinar, diagnosticar situações e formular soluções para problemas.

Salum NC e Prado ML (2014), afirma que as três habilidades requerem competências pessoais distintas, as quais traduzem qualidades de quem é capaz de analisar uma situação, apresentar soluções e resolver os assuntos ou problemas, constituindo sem capital intelectual. Moreira FG, et al. (2014), as atribuições do enfermeiro na atenção básica são essenciais na realização entre os usuários e famílias cadastradas nas equipes e, quando Indicado ou necessário, no domicílio e/ou nos demais espaços comunitários (escolas, associações etc.), em todas as fases do desenvolvimento humano: infância, adolescência, idade adulta e terceira idade, necessários para o adequado funcionamento da UBS.

Para Silva RNA, et al. (2016), a APS atualmente é a principal estratégia de promoção da saúde em âmbito individual e coletivo. Neto FRGX, et al. (2016), ressalta que a institucionalização do SUS dá se início a um intenso processo de descentralização e implantação de novas políticas, programas, projetos, serviços e ações de saúde nas três esferas de governo. Ou seja, vários programas articulados para promover melhorias relacionadas à saúde em cada comunidade.

Para Viacava F, et al. (2018), comenta que o SUS passou por algumas transformações durante a sua construção, entretanto os desafios ainda permanecem decorrente da APS e as suas transformações. Ainda assim, a presença de diversos atores públicos (Municipal, Estadual e Federal) empresas privadas, organizações não governamentais, cada uma delas subordinado a ambientes institucionais diferentes, iram proporciona orientações diversas.

O SUS ainda passar por muitas limitações seja nas quantidades, seja nas características dos serviços ofertados. A realidade atual do sistema de saúde público no Brasil não condis com a nossa realidade dos serviços prestados como a falta de insumos e leitos e profissionais capacitados nas redes de serviços ainda é bastante precário. Neste meio tempo, a ESF veio garantindo o espaço do enfermeiro e traçando algumas modalidades de intervenções, no modo de agir e pensar dos nossos representantes de saúde pública. A ESF tem o intuito de promover uma educação em saúde, visando orientar e capacitar os profissionais de saúde com a finalidade de fortalecer as ações ESF e aumentar a resolutividade da atenção à saúde no SUS (SILVA HP, CAXIAS MCL, 2016).

\section{CONSIDERAÇÕES FINAIS}

A atuação do enfermeiro na APS é um campo amplo e vasto em processo de qualificação, sejam na prática clínica ou gerencial, essas atividades laborais realizadas pelo enfermeiro na UBS são bastante relevantes, pois é possível identificar lacunas que precisam ser trabalhadas, dessa forma tornando-se fundamental para uma assistência de qualidade. O enfermeiro na APS tem a possibilidade de ampliar a sua autonomia por meio de uma prática clínica sustentada através de relações de diálogos, escuta ativa e praticando a humanização na perspectiva da integralidade e do cuidado às famílias e comunidades, refletindo na qualidade de vida da população, assim fortalecendo o vínculo do profissional e usuário, inovando nas ações desenvolvidas pela equipe de enfermagem, elaborando planos para as ações práticas utilizando a SAE, para desenvolver as suas atividades e gerenciamento.

\section{REFERÊNCIAS}

1. ACIOLI S, et al. Práticas de cuidado: o papel do enfermeiro na atenção básica [Nurses' work with children with cancer: palliative care]. Revista Enfermagem UERJ, 2014; 22(5): 637-642.

2. ANJOS KF, et al. Perspectivas e desafios do núcleo de apoio à saúde da família quanto às práticas em saúde. Saúde em Debate, Rio de Janeiro. 2013; 37(99): 672-680.

3. ARANTES LJ, et al. The benefits and challenges of the Family Health Strategy in Brazilian Primary Health care: a literature review. Ciencia \& saude coletiva; 2016; 21(5): 1499-1510. 
4. BEZERRA EAF, ALMEIDA JJJ. O papel do enfermeiro na promoção à saúde do homem: o contexto das unidades básicas de saúde da cidade de Macaíba/RN. SANARE-Revista de Políticas Públicas, 2014; 13(2).

5. BORGES F, et al. Performance of nurses in the bed management service of a teaching hospital. Rev. Bras. Enferm, 2020; 73 (4):20190349.

6. CAMARGO RAA, et al. Estratégia saúde da família nas ações primárias de saúde ao portador de hipertensão arterial sistêmica. Revista Mineira de Enfermagem, 2013; 17(4): 864-881.

7. CERVI GH, et al. Design of a Hospital Simulation Software for Nursing Education. In: Nursing Informatics. 2016; 995996.

8. DONIEC K, et al. A austeridade ameaça a cobertura universal de saúde no Brasil. The Lancet. 2016; 388(10047): 867868.

9. DUARTE E, et al. 30 anos do Sistema Único de Saúde. Epidemiol. Serv. Saúde, Brasília. 2018; 27(1): e00100018.

10. FERREIRA SRS, et al. The complexity of the work of nurses in Primary Health Care. Revista brasileira de enfermagem. 2018; 71: 704-709.

11. FERTONANI HP, et al. Modelo assistencial em saúde: conceitos e desafios para a atenção básica brasileira. Ciência \& Saúde Coletiva. 2015; 20: 1869-1878.

12. FIRMINO AA, et al. Atuação de enfermeiros na estratégia de saúde da família em um município de minas gerais. Saúde (Santa Maria). 2016; 42(1): 49-58.

13. FORTUNA CM, et al. Enfermagem em Saúde Coletiva: desejos e práticas. Rev. Bras. Enferm. Brasília, 2019; 72(1): 336-340.

14. KAHL C, et al. Acciones e interacciones en la práctica del enfermero en la Atención Primaria de Salud. Revista da Escola de Enfermagem da USP. 2018; 52.

15. KEBIAN LVA, ACIOLI SA. A visita domiciliar de enfermeiros e agentes comunitários de saúde da Estratégia Saúde da Família. Revista Eletrônica de Enfermagem. 2014;16(1): 161-9.

16. MACINKO J, HARRIS MJ. Brazil's family health strategy-delivering community-based primary care in a universal health system. N Engl J Med. 2015; 372(23): 2177-81.

17. MENDES EV. 25 anos do Sistema Único de Saúde: resultados e desafios. Estudos avançados. 2013; $27(78): 27-34$.

18. MELO PMJ. O planejamento estratégico nas ações em saúde e no trabalho do enfermeiro do programa de saúde da família. Revista Eletrônica Ciências Empresarias. 2018; 2(4): 33-62.

19. MOREIRA FG, et al. Capital intelectual como vantagem competitiva: um estudo bibliográfico. Revista de Carreiras. $2014 ; 4(3)$

20. MOROSINI MVGC, et al. Política Nacional de Atenção Básica 2017: retrocessos e riscos para o Sistema Único de Saúde. Saúde em Debate. 2018; 42: 11-24.

21. NEGRINI LDO, et al. Integração Ensino-Serviço-Comunidade: A Experiência Da Unidade De Saúde Escola Estratégia De Saúde Da Família" São Francisco De Assis" No Município De Bragança Paulista/Sp. Revista Ensaios Pioneiros. 2017; 1(1): 26-44.

22. NETO FRGX, et al. Necessidades de qualificação, dificuldades e facilidades dos técnicos de enfermagem na estratégia saúde da família. Sanare-Revista de políticas públicas. 2016;15(1).

23. NOGUEIRA IS, et al. Atuação do enfermeiro na atenção primária à saúde na temática do câncer: do real ao ideal. Revista de Pesquisa: Cuidado é Fundamental, 2019; 725-731.

24. OLIVEIRA SA, et al. Ferramentas gerenciais na prática de enfermeiros da atenção básica em saúde. Revista de Administração em Saúde. 2017; 17(69).

25. PAULA M, et al. Características do processo de trabalho do enfermeiro da estratégia de saúde da família. Revista Mineira de Enfermagem. 2014; 18(2): 454-470.

26. ROECKER S, et al. The educational work of nurses in the Family Health Strategy. Texto \& Contexto-Enfermagem. 2013; 22(1): 157-165.

27. SALUM NC, PRADO ML. A educação permanente no desenvolvimento de competências dos profissionais de enfermagem. Texto \& Contexto-Enfermagem. 2014; 23(2): 301-308.

28. SILVA RNA, et al. Conhecimento e entendimento de enfermeiros sobre as ações gerenciais na atenção primária à saúde. Ciência \& Saúde, 2016; 9(1): 21-29.

29. SILVA S, CARMO F. Princípio da Integralidade e os desafios de sua aplicação em saúde coletiva. Revista Saúde e Desenvolvimento. 2016; 7(4): 94-107.

30. SILVA SH, et al. Os diferentes olhares sobre humanização da assistência na Atenção Básica à Saúde. Revista de Educação Popular. 2020; 19(1): 45-63.

31. SOLER ZASG. Há litigância de má fé na atuação do enfermeiro como profissional liberal no Brasil? Enfermagem Brasil. 2018; 17(3):173-174.

32. STOPA SR, et al. Acesso e uso de serviços de saúde pela população brasileira, Pesquisa Nacional de Saúde 2013. Revista de Saúde Pública. 2017; 51(3s).

33. TEIXEIRA LA, et al. Mental health needs of adolescents and the nursing cares: integrative review. Texto \& contexto enferm, 2020; 29: e20180424.

34. VIEGAS SMF, PENNA CMM. O SUS é universal, mas vivemos de cotas. Ciência \& Saúde Coletiva. 2013; 18(1): 181190.

35. VIACAVA, Francisco et al. SUS: oferta, acesso e utilização de serviços de saúde nos últimos 30 anos, 2018 ; 23 (6): 1751-1762.

36. SILVA HP, CAXIAS MCL. Benefícios para alguns, prejuízos para muitos: razões e implicações da adoção da dupla porta de entrada em hospitais universitários. Saúde e Sociedade, 2016; 25: 808-820. 